\title{
Observing cross-sectional images and averaged optical patterns of photonic crystal fibers using partially incoherent laser light
}

\author{
F. W. Sheu \\ fwsheu@mail.ncyu.edu.tw
}

\section{J. Y. Chen}

\begin{abstract}
Department of Electrophysics, National Chiayi University, Chiayi 60004, Taiwan Graduate Institute of Optoelectronics and Solid State Electronics, National Chiayi University, Chiayi 60004, Taiwan

Graduate Institute of Optoelectronics and Solid State Electronics, National Chiayi University, Chiayi 60004, Taiwan
\end{abstract}

We couple the coherent laser light and the partially incoherent laser light into the photonic crystal fibers, respectively, and compare the observed fiber cross-sectional images. Lower coherence light can indeed help us obtain higher-quality and uniform cross-sectional images on the fiber output endfaces. We have observed rather unique and clear averaged optical mode patterns within the microstructures of a silica-air index-guiding solid-core photonic crystal fiber, which might be due to the stress-induced refractive-index inhomogeneity formed therein during the fiber manufacturing process. The experimental results are also compared with those images taken by other light sources or imaging systems. Besides, we successfully achieve capturing consecutively the various near-field averaged optical patterns of the solidcore photonic crystal fiber using this single-wavelength partially incoherent laser light imaging system.

[DOI: http://dx.doi.org/10.2971/jeos.2013.13003]

Keywords: Coherence, fiber imaging, photonic crystal fiber

\section{INTRODUCTION}

In a previous paper [1], we have reported that when a laser beam was launched into a multimode optical fiber, the optical pattern at the fiber output endface would exhibit fragmented light spots due to the field interference of higher-order modes. We controlled and reduced the optical coherence of the incident laser beam to a lower degree by placing a rotating diffuser [2] at the focus nearby of a focused laser beam. The fiber cladding became brighter and smoother due to the diffraction and penetration of the scattered fluctuating tiny light spots which were coupled into the fiber. Thus we could obtain a cross-sectional image of higher brightness and uniformity for the multimode fiber.

By transforming the input laser light into a partially incoherent laser light source, we can enhance the output beam uniformity as well as improve the fiber cross-sectional imaging $[1,3]$. This approach can also improve the fiber index profiling [4]. In addition, it provides an effective and practical method for improving the ultraviolet laser photolithography fabrication quality of microstructures [5]. Besides, through the partially incoherent stroboscopic light imaging technique, relatively high precision of in-plane vibration characterization of the microelectromechanical systems can also be achieved [6].

In this paper, we report a simplified configuration in which the laser beam is launched directly on a rotating diffuser [3] to obtain a partially incoherent laser light beam without the need of expanding and focusing the laser beam [1]. Besides, we turn to capture the cross-sectional images and averaged optical patterns of photonic crystal fibers (PCFs) [7, 8] which have attracted many research interests [9]-[17]. The original coherent speckled light spots at the output endface of PCFs could be smeared out efficiently by putting a rotating diffuser on the laser beam. The transformed partially incoherent laser light source can be used to obtain higher-quality crosssectional images of PCFs in comparison with a coherent laser light source. The probable stress-induced refractive-index inhomogeneity within the holey inner cladding and the central solid core of a silica-air index-guiding solid-core PCF can also be revealed distinctly by the observed averaged optical patterns. The detailed experimental results and their explanation will be demonstrated and discussed in the following two sections. In addition to improving the brightness and uniformity of the fiber cross-sectional images, the demonstrated partially incoherent laser light imaging system has a unique benefit in observing the various near-field averaged optical patterns of the PCF microstructures at a single optical wavelength in comparison with other light sources or imaging systems, which will be illustrated in the fourth and the fifth sections. Another technique of piezoelectric-based optical fiber spatial mode scrambling [18] can also be used to improve the beam uniformity, but it can deform the microstructures of the test PCF fast and randomly, prohibiting the observation of the unique optical mode patterns that probably result from the stress-induced refractive-index inhomogeneity formed within the microstructures during the PCF manufacturing process. Therefore our proposed imaging system using the partially incoherent laser light has the benefits of inspecting the inhomo- 
geneous refractive-index variation within the photonic crystal microstructures and observing conveniently the various nearfield averaged optical mode patterns from the captured fiber images of higher clearness and uniformity.

\section{PCF CROSS-SECTIONAL IMAGING BY COHERENT LASER LIGHT}

The experimental setup used in this section is shown in Figure 1 . We focus a continuous-wave linearly polarized coherent He-Ne laser light beam (wavelength $=632.8 \mathrm{~nm}$ ) by a 20X microscope objective (MO) lens into a test fiber, which is mounted on a three-dimensional translation stage. To observe the fiber cross-sectional image and optical mode pattern, the transmitted light from the output endface of the test fiber is projected onto a CCD camera (Newport, LBP-3-USB) through another 10X MO which acts as an imaging lens. We take a solid-core PCF (Blazephotonics, ESM-12-01) and a hollowcore PCF (Blazephotonics, HC-800-1) to test the mode patterns, respectively. The experimental results are shown in Figure 2 .

The solid-core PCF specification: solid core diameter $\approx 12 \mu \mathrm{m}$, holey region diameter $\approx 60 \mu \mathrm{m}$, outside diameter $\approx 125 \mu \mathrm{m}$, pitch spacing $\approx 8 \mu \mathrm{m}$, hole diameter $\mathrm{d} \approx 3.68 \mu \mathrm{m}$, fiber length $\approx 6 \mathrm{~cm}$. The hollow-core PCF specification: hollow core diameter $\approx 9 \mu \mathrm{m}$, holey region diameter $\approx 40 \mu \mathrm{m}$, silica cladding diameter $\approx 135 \mu \mathrm{m}$, pitch distance $\approx 2.3 \mu \mathrm{m}$, air filling fraction $>90 \%$, fiber length $\approx 6 \mathrm{~cm}$. Figures $2(\mathrm{a})$-(b) show the observed cross-sectional images of the solid-core PCF, and Figures 2(c)-(d) show those of the hollow-core PCF. The observed fiber images will change when the fiber coupling position of the coherent laser light is laterally adjusted from the inner cladding to the outer cladding. Figures 2(a) and 2(c) show holey-disk-shaped inner cladding modes of which the laser light is guided by the numerous effective secondary cores of the glass substrands full of many air holes [14]. Figures 2(b) and 2(d) show donut-shaped outer cladding modes [14]. Since the refractive index of the solid outer cladding is higher than the averaged index value of the holey inner cladding, the outer cladding is also an effective waveguide which can confine the light within it. Nevertheless, as the laser light of high optical coherence is launched into the fiber, all the observed optical patterns exhibit fragmented optical speckles due to the field interference of higher-order modes. As a consequence, the imaging system which uses the coherent laser light as the light source cannot be exploited to observe high-quality fiber cross-sectional images and uniform optical mode patterns within the photonic crystal microstructures.

\section{PCF CROSS-SECTIONAL IMAGING BY PARTIALLY INCOHERENT LASER LIGHT}

To overcome the difficulties in the fiber cross-sectional imaging by coherent laser light, we try to reform the laser light source by reducing its optical coherence level. Figures 3(a) and 3(b) show the experimental setup used to transform the incident coherent laser light into a partially incoherent laser light source and demonstrate how to couple this low coher- (a)
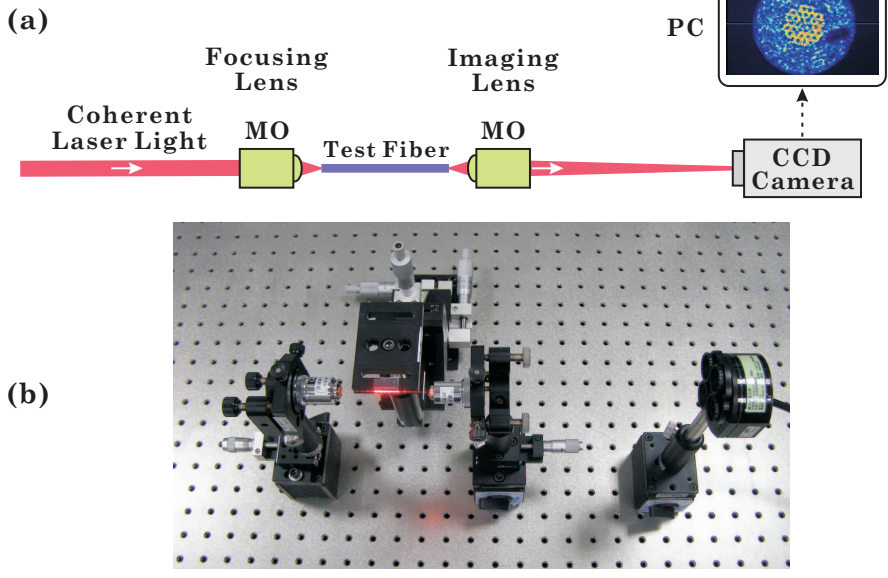

FIG. 1 The (a) configuration and (b) photograph of the experimental setup for coupling a coherent laser light beam into a test fiber and observing the fiber cross-sectional image. MO, microscope objective; PC, personal computer; CCD, charge-coupled device. (a)

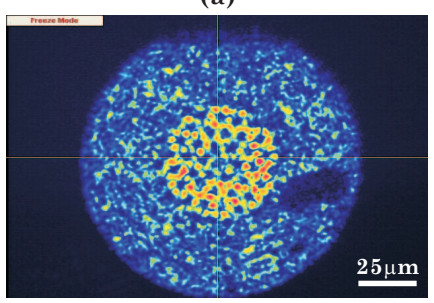

(c)

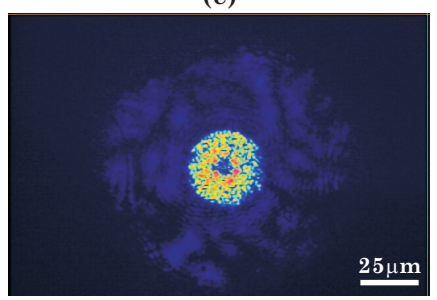

(b)

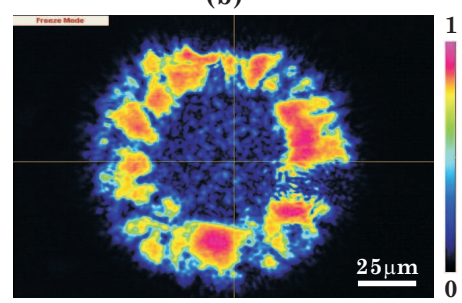

(d)

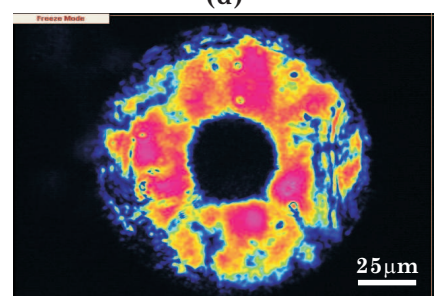

FIC. 2 The observed cross-sectional images of (a)-(b) the solid-core PCF and (c)-(d) the hollow-core PCF when the fiber coupling position of the coherent laser light is laterally adjusted from the inner cladding to the outer cladding.

ence light beam into the test fiber. We first let the He-Ne laser beam pass directly through a semi-transparent diffuser which can be driven by a fast rotating motor [3]. After transmitting the diffuser, the laser beam is scattered into many tiny light spots (Figure 3(c)), which behave as many new point light sources. When the diffuser is rotating, all the light spots will move and change randomly such that the averaged light intensity appears to be smoothly distributed (Figure 3(d)) and statistically stationary. Nevertheless, the optical coherence of the scattered fluctuating light beam will decrease as a consequence of the instantaneous highly fragmented beam shape. The rotation frequency of the diffuser in the experiments is $30-50 \mathrm{~Hz}$ approximately. The resultant averaged duration of the tiny light spots among the highly fragmented and fast fluctuating laser beam is much smaller than the response time of the CCD camera. If the rotation speed of the diffuser cannot be increased any more due to mechanical instability, in order to further increase the fiber image uniformity, the captured image can be produced alternatively by averaging tens of snapshot images, which can be achieved by adjusting the image acquisition softwares average setting or by performing 


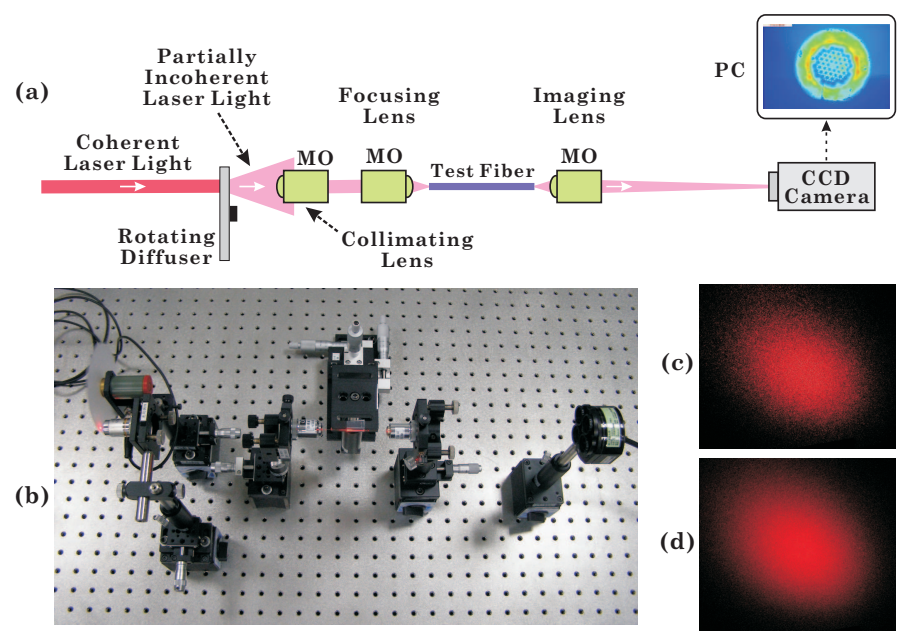

FIG. 3 The (a) configuration and (b) photograph of the experimental setup for coupling a partially incoherent laser light beam into a test fiber and observing the fiber crosssectional image. The observed far-field optical speckles just behind the diffuser when the diffuser is (c) stopped and (d) rotating.

the calculation with a mathematical software. The main action of the diffuser is to fragment the laser beam into many tiny light spots and to decrease the degree of spatial coherence of the laser light. The rotation speed of the diffuser somewhat affects the degree of temporal coherence (or coherence time) of the transformed laser light. However the degree of temporal coherence of the transformed laser light is still rather high under various achievable rotation speeds of the diffuser, since the rotation speed of the diffuser is far smaller than the propagation speed of the light. The rotation speed of the diffuser mainly affects the fiber image uniformity, which is also related to the characteristics of the used CCD camera. The resultant partially incoherent laser light source will be shown to be capable of improving the quality of the fiber cross-sectional images and increasing the uniformity and clearness of the optical mode patterns.

Furthermore, as shown in Figure 3(a), we use the first MO (10X) to collimate the central part of the scattered diffracting light beam, and then use the second MO (20X) to focus and couple the collected partially incoherent laser light into the test fiber. Finally, the cross-sectional image at the fiber output endface is projected onto a CCD camera by the third MO (10X).

The observed cross-sectional images of the solid-core PCF ESM-12-01 and the hollow-core PCF HC-800-1 using the partially incoherent laser light are shown in Figure 4 . The observed fiber cross-sectional images and the optical mode patterns turn to be smoother and clearer due to the rotation of the diffractive diffuser. In addition, as shown in Figures 4(a) and 4(c), for the solid-core PCF with a triangular lattice of air holes, we can observe the similar hexagonal-latticed inner cladding mode $[8,13]$. However, instead of a fundamental mode in the central solid core, we have observed a sixlobed solid-core mode $[8,16]$, which is attributed to the probable stress-induced inhomogeneous refractive-index variation formed at the central solid-core region and caused by the compressive stress from the surrounding hexagonal-latticed air holes during the fusion, drawing and cooling process (a)

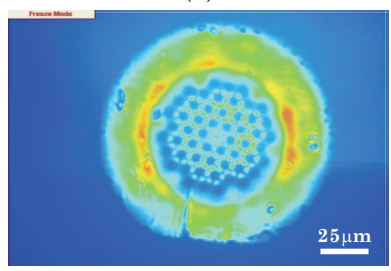

(c)

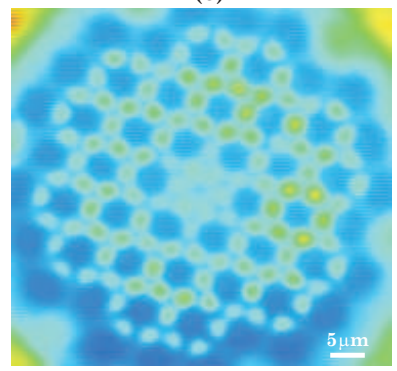

(b)

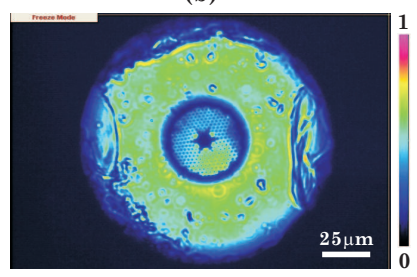

(d)

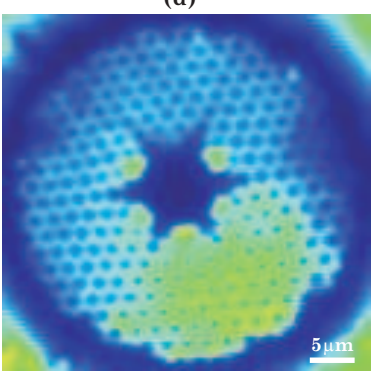

FIC. 4 The observed cross-sectional images of (a) the solid-core PCF and (b) the hollowcore PCF when the partially incoherent laser light is coupled into the fiber. (c) and (d) are the cut and enlarged images of (a) and (b) at the holey inner cladding regions, respectively.

of silica micro tubes and rods. The six-lobed central solidcore mode might also be due to the reason that the normalized air hole diameter $d / \Lambda$ for the solid-core PCF ESM-12-01 has a value of 0.46 approximately, exceeding the endlessly single-mode regime for a solid-core PCF [19]-[29] under the $632.8 \mathrm{~nm}$ He-Ne laser wavelength. The resultant higher-order modes will dominate over the fundamental mode in the central solid core under a probable stress-induced inhomogeneous refractive-index variation with hexagonal symmetry, consequently forming a six-lobed averaged optical mode pattern therein.

We can obtain a higher-quality, clear and uniform fiber crosssectional image for the PCF using this partially incoherent laser light imaging system, overcoming the encountered difficulty when using the coherent laser light imaging system. Nevertheless, as shown in Figures 4(b) and 4(d), the captured cross-sectional image around the central major air hole of the hollow-core PCF HC-800-1 exhibits star-light-like darkness with six sharp capes, which might be due to the six-fold symmetry arrangement in the size difference of the surrounding minor air holes. In addition, no $632.8 \mathrm{~nm}$ He-Ne laser light can be guided in the hollow core of the PCF HC-800-1 (Figures $4(\mathrm{~b})$ and $4(\mathrm{~d})$ ), since the nominal center operating wavelength of the guided modes in the hollow core of the PCF $\mathrm{HC}-800-1$ is $830 \mathrm{~nm}$.

From another point of view, as shown in Figure 4, unlike the technique of a certain mode excitation [10,14], our proposed imaging technique using the partially incoherent laser light can excite many higher-order modes simultaneously in the solid outer cladding of the PCFs, and thus we can obtain a bright and clear averaged cross-sectional image in the solid outer cladding by fast moving all the fragmented tiny light spots with the rotating diffuser. However, to our surprise, the observed averaged cross-sectional image in the holey inner cladding of the solid-core PCF (Figure 4(c)) exhibits a very unique hexagonal-latticed optical pattern with multiple reg- 
ular light spots discretely distributed in the novel effective secondary cores of the glass substrands. Each of the statistically stationary light spots in the holey inner cladding is found to be situated at the midpoint of every two neighboring air holes, rather than at the center of every three neighboring air holes as described in [8, 13]. We also attribute this phenomenon to the probable stress-induced refractive-index inhomogeneity within the PCF microstructures during the fusion, drawing and cooling process of silica micro tubes and rods. The statistically averaged optical pattern in Figure 4(a) due to the superposition of many fast fluctuating tiny light spots in the holey inner cladding of the solid-core PCF looks more uniform and more distinct than the discrete optical pattern in Figure 2(a) captured with coherent laser light, of which the apparently random many-spot pattern also shows implicitly the probable stress-induced inhomogeneous index increasing and the resultant light guiding at the middle of every two neighboring air holes. Besides, the little refractiveindex difference between the fiber core and the fiber cladding is only approximately 0.005 for a conventional single-mode optical fiber. Hence it is a reasonable conjecture to attribute the observed optical patterns to the probable stress-induced refractive-index variations between every two neighboring air holes in the holey inner cladding of the solid-core PCF. The similar phenomenon is also illustrated in [17]. The authors therein present a novel air-silica structured index-guiding holey fibers with an annulus mode profile by introducing a new ring defect design. The new defect consists of a central air hole and a germanosilicate ring surrounding it, which results in a large-area annulus mode profile. When the fiber was drawn in a higher temperature or lower internal pressure, the ring defects collapsed to result in solid multiple high index solid cores. In this case, the modal shape is no longer annular but becomes highly compatible with that of the $\mathrm{LP}_{01}$ mode of conventional single-mode fibers [17]. In our experiments, in fact, the light guiding by the stress-induced refractive-index inhomogeneity within the holey inner cladding and the central solid-core region of the solid-core PCF has been revealed implicitly by the coherent laser light fiber cross-sectional image (Figure 2(a)). This phenomenon can be further identified more clearly by the partially incoherent laser light fiber crosssectional images (Figures 4(a) and 4(c)).

On the other hand, the observed averaged cross-sectional image in the holey inner cladding of the hollow-core PCF (Figure $4(\mathrm{~d})$ ) exhibits a holey continuous optical pattern with multiple connected meshes uniformly distributed within all the highly tiny glass substrands, proving that the proposed technique in this study can be used to capture high resolution cross-sectional images of the PCF microstructure with smaller effective mode area.

The wavelength dependence of the guided mode patterns of PCFs could be measured by the dispersive monochromatic light of an incoherent broadband tungsten lamp or supercontinuum light source $[8,10]$, or by a wavelength tunable laser source [14]. The above cited techniques can also excite a certain mode of the PCF by adjusting the coupling condition (the numerical aperture of the used $\mathrm{MO}$ ) at the fiber input end. Nevertheless, according to our experiences in this study, when using a wavelength tunable laser source, if one would like to observe clearly the fiber cross-sectional images of PCFs at a certain single wavelength, the coherent laser light beam must be transformed into a partially incoherent laser light beam before being launched into the test fiber by the use of a rotating diffuser or a similar apparatus. This treatment can greatly improve the quality of the captured averaged optical pattern of a test PCF, which consists of many fast fluctuating tiny light spots.

\section{PCF CROSS-SECTIONAL IMAGING BY OTHER LIGHT SOURCES AND IMAGING SYSTEMS}

In this section, we will explore and compare the crosssectional imaging effects of PCFs by other light sources or imaging systems [3]. Figures 5(a) and 5(b) show the observed cross-sectional images of the solid-core PCF and the hollowcore PCF when using the incoherent light-emitting diode (LED) white light as the light source instead in the system of Figure 3(a). The stress-induced six-lobed mode in the central solid core of the PCF ESM-12-01 (Figure 5(a)) can still be observed even if the incident LED white light is broadband, because it can deliver the broadband radiation in a single spatial mode [8]. No incoherent LED white light can be guided in the hollow core of the PCF HC-800-1 (Figure 5(b)) just like the case of the $632.8 \mathrm{~nm}$ He-Ne laser partially incoherent light imaging system (Figure 4(b)), since the nominal center operating wavelength of the guided modes in the hollow core of the PCF HC-800-1 is $830 \mathrm{~nm}$. Nevertheless, the glass substrands in the holey inner cladding and the ring-shaped solid outer cladding are effective waveguides, (a)

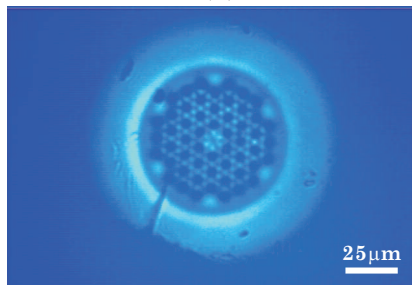

(c)

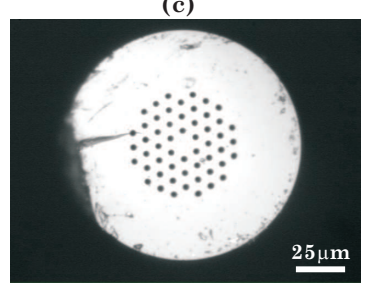

(e)

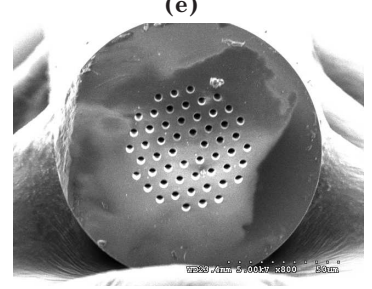

(b)

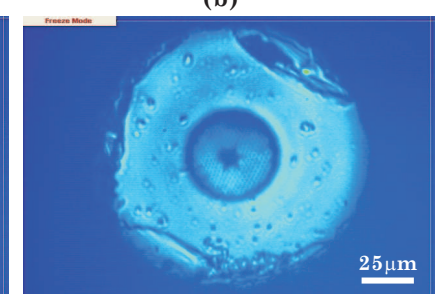

(d)

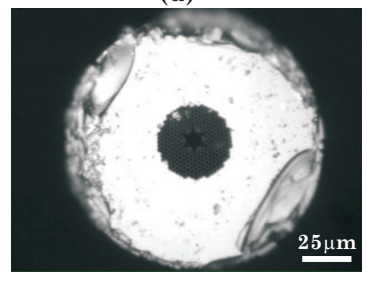

(f)

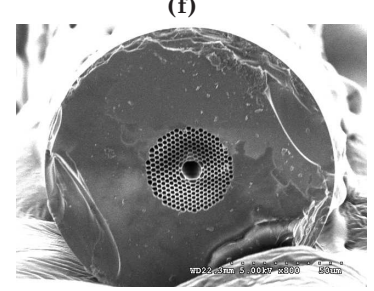

FIG. 5 The observed cross-sectional images of the solid-core PCF (left column) and the hollow-core PCF (right column): (a)-(b) when the incoherent LED white light is coupled into the fiber, (c)-(d) when using the reflective white-light $0 \mathrm{M}$, and (e)-(f) when using the SEM. 
and thus they can guide the LED white light just like the case of the He-Ne laser partially incoherent light imaging system (Figure 4(b)). The drawback of the used incoherent LED white light imaging system is that the optical power coupled into the fiber is rather low and inhibit the brightness of the fiber images, which could be improved by replacing the original LED device with a high-power LED.

The reflective white-light optical microscope (OM, Navitar) is a traditional tool to observe the micrographs. Figures 5(c) and 5(d) show the observed cross-sectional images of the solidcore PCF and the hollow-core PCF using the incoherent halogen lamp white light. Limited by the depth of focus of the 50X MO, the fiber image captured by the reflective whitelight OM cannot reveal clearly the stereo configuration of the fiber endface. Another drawback is that the reflected light of the tiny microstructure in the holey inner cladding region of the hollow-core PCF is very weak such that it looks rather dark (Figure 5(d)). Besides, it cannot be used to observe the optical mode patterns of PCFs. A scanning electron microscope (SEM, Hitachi) is also utilized to inspect the stereo micrographs of the solid-core PCF and the hollow-core PCF as shown in Figures 5(e) and 5(f). The expensive and sophisticated SEM imaging system can provide the most detailed and highest-quality fiber cross-sectional images, but it also cannot be used to observe the optical mode patterns of PCFs. These examples illustrate the advantage of using the proposed single-wavelength partially incoherent laser light imaging system to explore the averaged optical mode patterns of PCFs and inspect the probable stress-induced refractive-index inhomogeneity within the photonic crystal microstructures.

\section{OBSERVING THE CONSECUTIVE VARIATION IN THE PCF NEAR-FIELD AVERAGED OPTICAL PATTERN}

As shown in Figure 4(a), higher-quality fiber cross-sectional images and specific averaged optical mode patterns of a solidcore PCF can be obtained as we couple the partially incoherent laser light into the fiber. While translating the imaging lens (Figure 3(a)) slightly in the longitudinal direction, we find that we can also achieve capturing the various near-field averaged optical patterns of the solid-core PCF using the He-Ne laser partially incoherent light imaging system. As shown in Figure 6 , the near-field diffraction images of the transmitted light from the fiber output endface $[12,30]$, through adjusting consecutively the focusing plane of the imaging lens, can display progressively the quasi-apex mode, the quasi-strut mode, and the quasi-airy mode [13] of the novel effective secondary cores with six-fold hexagonal symmetry in the holey inner cladding region. Compared to the scanning near-field optical microscope (SNOM) which uses a fiber tip to scan and collect the intensity distribution information of the imaged transverse mode [11, 13], the imaging system using the partially incoherent laser light provides a more simple, fast, and convenient measurement method.

The image contrast in Figure 6 was not tuned to a higher value similar to that in Figure 4(a) while recording the movie. In fact, it can be controlled by adjusting the distance between the col- (a)

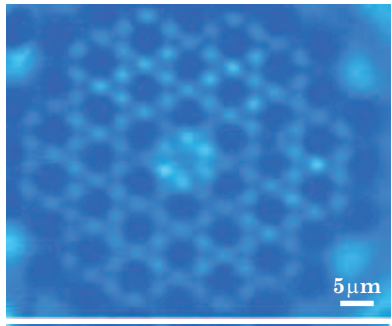

(b)

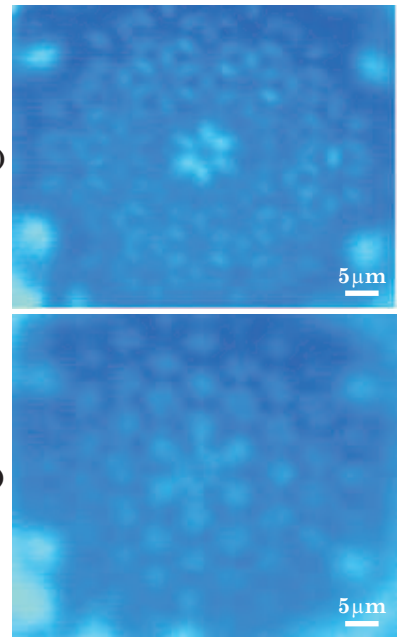

FIG. 6 The observed various near-field averaged optical patterns (videosize: $3.21 \mathrm{MB}$, format: mov, see Fig6.mov) at the neighborhood of the output endface of the solidcore PCF: (a) the quasi-apex mode, (b) the quasi-strut mode, and (c) the quasi-airy mode in the holey inner cladding region when the partially incoherent laser light is coupled into the fiber.

limating lens and the focusing lens (Figure 3(a)), by changing the attenuation filter in front of the CCD camera, or by resetting the shutter speed or gain control on the image acquisition software. We have not noticed this problem until we have taken the SEM images of the PCFs. The metal coating on the PCF surface during the SEM imaging process inhibits us from performing again the fiber cross-sectional optical imaging on the original test PCF. We therefore did not record the movie again at a higher image contrast.

\section{CONCLUSION}

We have successfully measured the higher-quality crosssectional images and very unique averaged optical mode patterns of PCFs using the partially incoherent laser light. We have also compared the observed fiber images with those taken by other light sources or imaging systems. Besides, we also successfully achieve observing the consecutive variation in the near-field averaged optical pattern at the neighborhood of the output endface of the solid-core PCF using this singlewavelength partially incoherent laser light imaging system. This simple approach can efficiently improve the brightness and uniformity of the fiber cross-sectional images and also provide an excellent solution for the investigation on the averaged optical patterns inside (in the cross-section) or outside (in the near-field) the PCF microstructures. The experimental measurement results can provide us with useful and valuable information about the probable existence and placement of the stress-induced refractive-index inhomogeneity formed within the microstructures of a silica-air index-guiding 
solid-core PCF during the fiber manufacturing process. The characterization of PCFs using the partially incoherent laser light can also advance more physical insight into the optical coherence and the photonic crystal optical waveguide.

\section{ACKNOWLEDGEMENTS}

We acknowledge the financial support partly from the National Science Council, Taiwan, through Projects NSC 95-2112-M-415-004 and NSC 100-2112-M-415-002-MY3, and partly from the National Chiayi University, through Project NCYU 97T001-05-04-001. We also gratefully acknowledge the Department of Physics, National Chung Cheng University, Taiwan, for the assistance in the SEM imaging of the PCFs.

\section{A SUPPLEMENTARY DATA}

Supplementary data associated with Figure 6 of this article can be found, in the online version, at doi:10.2971/jeos.2013. 13003 .

\section{References}

[1] F. W. Sheu and J. Y. Chen, "Fiber cross-sectional imaging by manually controlled low coherence light sources," Opt. Express 16, 22113-22118 (2008).

[2] M. Mitchell, Z. Chen, M. F. Shih, and M. Segev, "Self-Trapping of Partially Spatially Incoherent Light," Phys. Rev. Lett. 77, 490-493 (1996).

[3] F. W. Sheu, and J. Y. Chen, "Observing cross-sectional images of various optical fibers using low coherence transformed laser light," Phys. Procedia 19, 325-328 (2011).

[4] W. S. Tsai, W. S. Wang, and P. K. Wei, "Two-dimensional refractive index profiling by using differential near-field scanning optical microscopy," Appl. Phys. Lett. 91, 061123 (2007).

[5] F. W. Sheu, C. Y. Weng, C. H. Chen, "Improved fabrication of circular-grating microstructured devices using partially spatially incoherent ultraviolet laser light," Opt. Commun. 285, 2990-2995 (2012).

[6] D. A. Wang, F. W. Sheu, Y. S. Chiu, "In-plane vibration characterization of microelectromechanical systems using acousto-optic modulated partially incoherent stroboscopic imaging," Opt. Laser. Eng. 49, 954-961 (2011).

[7] P. St. J. Russell, "Photonic Crystal Fibers," Science 299, 358-362 (2003).

[8] P. St. J. Russell, “Photonic-Crystal Fibers," J. Lightwave Technol. 24, 4729-4749 (2006).

[9] N. Mortensen, and J. Folkenberg, "Near-field to far-field transition of photonic crystal fibers: symmetries and interference phenomena," Opt. Express 10, 475-481 (2002).

[10] G. Humbert, J. Knight, C. Bouwmans, P. Russell, D. Williams, P. Roberts, and B. Mangan, "Hollow core photonic crystal fibers for beam delivery," Opt. Express 12, 1477-1484 (2004).

[11] J. Gates, C. Hillman, Joanne Baggett, K. Furusawa, T. Monro, and W. Brocklesby, "Structure and propagation of modes of large mode area holey fibers," Opt. Express 12, 847-852 (2004).
[12] J. D. Shephard, P. J. Roberts, J. D. C. Jones, J. C. Knight, and D. P. Hand, "Measuring Beam Quality of Hollow Core Photonic Crystal Fibers," J. Lightwave Technol. 24, 3761-2769 (2006).

[13] F. Couny, F. Benabid, P. J. Roberts, M. T. Burnett, and S. A. Maier, "Identification of Bloch-modes in hollow-core photonic crystal fiber cladding," Opt. Express 15, 325-338 (2007).

[14] S. D. Lim, H. C. Park, I. K. Hwang, S. B. Lee, and B. Y. Kim, “Experimental excitation and characterization of cladding modes in photonic crystal fiber," Opt. Express 18, 1833-1840 (2010).

[15] J. Broeng, S. E. Barkou, A. Bjarklev, J. C. Knight, T. A. Birks, P. St. J. Russell, "Highly increased photonic band gaps in silica/air structures," Opt. Commun. 156, 240-244 (1998).

[16] S. Konorov, E. Serebryannikov, A. Zheltikov, P. Zhou, A. Tarasevitch, and D. von der Linde, "Mode-controlled colors from microstructure fibers," Opt. Express 12, 730-735 (2004).

[17] S. Kim, Y. Jung, K. Oh, J. Kobelke, K. Schuster, and J. Kirchhof, "Defect and lattice structure for air-silica index-guiding holey fibers," Opt. Lett. 31, 164-166 (2006).

[18] L. Yan, X. S. Yao, L. Lin, and X. Chen, “Improved beam uniformity in multimode fibers using piezoelectric-based spatial mode scrambling for medical applications," Opt. Eng. 47, 090502 (2008).

[19] N. A. Mortensen, "Effective area of photonic crystal fibers," Opt. Express 10, 341-348 (2002).

[20] N. A. Mortensen, J. R. Folkenberg, M. D. Nielsen, and K. P. Hansen, "Modal cutoff and the V parameter in photonic crystal fibers," Opt. Lett. 28, 1879-1881 (2003).

[21] M. Nielsen, and N. Mortensen, "Photonic crystal fiber design based on the V-parameter," Opt. Express 11, 2762-2768 (2003).

[22] G. Renversez, F. Bordas, and B. T. Kuhlmey, "Second mode transition in microstructured optical fibers: determination of the critical geometrical parameter and study of the matrix refractive index and effects of cladding size," Opt. Lett. 30, 1264-1266 (2005).

[23] K. Saitoh, Y. Tsuchida, M. Koshiba, and N. A. Mortensen, “Endlessly single-mode holey fibers: the influence of core design," Opt. Express $13,10833-10839$ (2005).

[24] J. Liu, G. Kai, L. Xue, Z. Wang, Y. Liu, Y. Li, C. Zhang, T. Sun, and $X$. Dong, "Modal cutoff properties in germanium-doped photonic crystal fiber," Appl. Opt. 45, 2035-2038 (2006).

[25] X. Zhao, L. Hou, Z. Liu, W. Wang, G. Zhou, and Z. Hou, "Improved fully vectorial effective index method in photonic crystal fiber," Appl. 0pt. 46, 4052-4056 (2007).

[26] W. Chen, J. Li, S. Li, H. Li, Z. Jiang, and J. Peng, "Study on single-mode photonic crystal fibers in wide wavelength range," Chin. Opt. Lett. 5, 383-385 (2007).

[27] Y. Li, Y. Yao, M. Hu, L. Chai, and C. Wang, "Improved fully vectorial effective index method for photonic crystal fibers: evaluation and enhancement," Appl. Opt. 47, 399-406 (2008).

[28] M. Y. Chen, "All-solid silica-based photonic crystal fibers," Opt. Commun. 266, 151-158 (2006).

[29] C. Wang, F. Zhang, Y. Lu, C. Liu, R. Geng, T. Ning and S. Jian, "Single-mode operations in the large flattened mode optical fiber lasers and amplifiers," J. Opt. A: Pure Appl. Opt. 11, 065402 (2009).

[30] W. Ha, S. Lee, J. Kim, Y. Jeong, K. Oh, J. Kobelke, K. Schuster, S. Unger, A. Schwuchow, and J. K. Kim, "A micro-structured aperture made of a hollow triangular-core fiber for novel beam shaping," Opt. Express 18, 20918-20925 (2010). 